Edward, D. G. fF. \& Fitzgerald, W. A. (1951). J. gen. Microbiol. 5, 566-575.

\title{
The Isolation of Organisms of the Pleuropneumonia Group from Dogs
}

\author{
By D. G. Fr. EDWARD AND W. A. FITZGERALD \\ The Wellcome Veterinary Research Station, Frant, Sussex, and \\ The Wellcome Research Laboratories, Beckenham, Kent
}

\begin{abstract}
SUMMARY: The finding of a pleuropneumonia-like organism associated with infertility and epididymitis in a dog led to the examination of throat and vaginal swabs from all the animals in a breeding kennel. Pleuropneumonia-like organisms were isolated from the majority of samples. Strains differed in colonial appearances, and a classification into types based on these differences was confirmed by the serological demonstration that each type was antigenically distinct. Nearly all the strains belong to one of three types, provisionally called $\alpha, \beta$ and $\gamma$. Each type was regarded as a distinct species of the pleuropneumonia group, and its biological properties were studied.
\end{abstract}

The semen of a dog admitted for investigation of sterility and epididymitis was used for the artificial insemination of two bitches in the breeding kennel. Two days later a stud dog was mated with one of the bitches. Soon afterwards the semen of this dog showed evidence of impaired fertility and inflammation of the epididymis was suspected. A bacteriological examination of the semen yielded positive cultures of pleuropneumonia-like organisms. Similar organisms were cultivated from the semen of the dog originally brought for investigation and from a vaginal swab from the bitch which had been inseminated. These findings suggested that an organism of the pleuropneumonia group had been found which was pathogenic for the canine genital tract. With the idea of investigating the distribution of the organism, cultures were made from vaginal swabs or semen, and from throat swabs obtained from all the animals in the kennels. Pleuropneumonia-like organisms were isolated from the majority of specimens, but the cultural appearance of the strains varied so much as to suggest that several different members of the pleuropneumonia group inhabited the genital and respiratory tracts of these dogs. In only a few of the cultures did the colonies resemble those of the strains isolated originally from the two dogs and one bitch. The investigation described in this paper, which confirmed that the strains belonged to several different species of the pleuropneumonia group, enabled each strain to be identified. Unfortunately, however, owing to the time taken to accomplish this and to the difficulty in maintaining the many strains meanwhile, it was not possible to investigate pathogenicity.

\section{METHODS}

Vaginal swabs were taken through a glass speculum, after thorough cleansing of the external genitals with spirit. Cultures were made on a special selective medium (Edward, 1947). Two agar plates and one bottle of semi-solid medium were inoculated with each sample; one plate was incubated aerobically and the 
other anaerobically. The plates were examined after 3 and 4 days' incubation, using a binocular dissecting microscope $(\times 10)$ and slightly oblique transmitted light. When colonies of pleuropneumonia-like organisms were seen, subcultures were made from single colonies; on some plates there appeared to be more than one type of colony, and subcultures were made from each type.

For serological investigation suspensions of organisms were prepared from cultures in broth enriched with horse serum or human ascitic fluid. Antisera were made in rabbits by repeated inoculation with the deposits of cultures grown in rabbit serum broth. Certain strains did not grow in rabbit serum broth, but good growth was obtained when cholesterol $(0 \cdot 1 \mathrm{mg} . / \mathrm{ml}$.) was added to the medium as a finely dispersed suspension (Edward \& Fitzgerald, 1951). The techniques for the agglutination and complement-fixation tests were the same, except for a few modifications as those used previously in the study of bovine genital strains (Edward, $1950 a$ ). The agglutination tests were incubated at $52^{\circ}$ for $6 \mathrm{hr}$. and then at $37^{\circ}$ overnight, and readings were made at 4,6 and $20 \mathrm{hr}$. In the complement-fixation tests two full haemolytic units of complement were used.

\section{RESULTS}

\section{Isolation of strains}

Swabs from vagina and throat of thirty-nine bitches were examined; from only four animals were the cultures from both swabs negative for pleuropneumonialike organisms. From fifteen bitches, pleuropneumonia-like organisms were grown from both vagina and throat; from six, vaginal swabs were positive and throat negative, and from fourteen, throat was positive and vagina negative. The colonial appearances of the various strains differed markedly. These differences, first apparent in the primary culture, were maintained in the early subcultures. Some swabs yielded at least two types of colony, which bred true on subculture. These observations suggested that several different members of the pleuropneumonia group inhabited the throat and genital tracts of these dogs. In a preliminary classification of the strains based on their colonial appearances three types were recognized; for convenience they were designated by the first three letters of the Greek alphabet.

$\alpha$-Strains. These strains, although not the most numerous, were of special interest. Of this type were the three strains which had been isolated from the infertile dog, from the bitch mated with it and from the stud dog apparently infected by subsequent mating with the infected bitch. The $\alpha$-strains were distinguished by having a coarsely reticulated colony. When viewed by transmitted light the thick dark markings suggested the presence of large globules inside the colony (Pl. 1, fig. 1). In young colonies often only the centre was heavily marked, the periphery being conspicuously translucent with a smooth surface resembling a drop of water. The colonies were otherwise typical of the pleuropneumonia group, except that the central spot tended to be hidden by the coarse markings. Most of the $\alpha$-strains were isolated from semen or vaginal material. The colonies of these genital strains were small and about the same size as those of human and bovine genital strains. There were also a few strains, isolated from throats, whose colonies had similar markings, but were larger. 
$\beta$-Strains. These strains were the most common and were isolated from both throat and genital tract. The colonies were large and the central spot, which is so characteristic a feature of colonies of the pleuropneumonia group, was absent or poorly developed. These characters were most noticeable on primary isolation. On the original plates the colonies were so large, that, having no obvious central spot, it was difficult to recognize them as belonging to the pleuropneumonia group. However, they had the typical translucency, fine surface markings, and other characters of pleuropneumonia-like organisms (Pl. 1, fig. 2). Some strains, after 3 days' incubation, formed flattened colonies with irregular margins. With repeated subculture the special features characterizing the $\beta$-strains tended to disappear, more quickly with some strains than with others, so that they became indistinguishable from other members of the pleuropneumonia group.

$\gamma$-Strains. A number of strains, isolated from vagina and throat, when grown on media enriched with horse serum yielded surface growths which became covered by a peculiar 'pearly' film; these were termed ' $\gamma$ '-strains. Under the dissecting microscope the film was crinkled, covering the colonies and spreading between them. There were also numerous small black dots in the substance of the medium just beneath and around the colonies. These appearances were identical with those described in cultures of two other members of the pleuropneumonia group, the $\mathbf{P}$ organism from the bovine genital tract and the L3 organism from mice (Edward, 1950a, $b$ ). With the $\gamma$-strains, however, the film appeared earlier, often by the third day of incubation, and was better developed than with the other organisms. Colonies of the $\gamma$-strains otherwise had no distinctive characters (Pl. 1, fig. 3).

From one vaginal swab a pleuropneumonia-like organism was obtained on a plate incubated anaerobically, the corresponding aerobically incubated plate remaining sterile. Attempts at subculture aerobically and anaerobically failed. It was thus impossible to establish whether this strain was an obligatory anaerobe and different from the others.

Dienes $(1947 a, b)$ called attention to a possible danger in using penicillin to provide a selective medium for the isolation of pleuropneumonia-like organisms. Pleuropneumonia-like forms can be developed as variants from various bacteria on media containing penicillin. In view of the large number of strains isolated here from dogs by the use of media containing penicillin, it was necessary to exclude the possibility that the organisms had been derived from bacteria in the specimens taken. In making the cultures penicillin was always applied only to half of the agar plate. Both halves were inoculated, and it was noted that colonies of the pleuropneumonia-like organisms appeared on the penicillinfree part of the plate, except when they were overgrown or inhibited by bacteria. A number of strains which were subcultured directly on to media free from penicillin retained their characters and did not become bacillary in form even though subculture was repeated on penicillin-free media.

The preliminary three classes of strains, each possibly a different species of the pleuropneumonia group, were further investigated by cultural and serological methods. In order to decrease the number of strains to be examined, 
strains with colonies exactly similar to those of another strain were discarded. There remained thirty-four strains from which suspensions were prepared for serological study. Typical $\alpha$-, $\beta$ - and $\gamma$-strains were included, together with strains whose identification on colonial appearance was doubtful. The serological investigation, which provided most information and confirmed the original classification, is reported first.

\section{Serological investigation}

Antisera were prepared in rabbits against two representative $\alpha$-strains, strain no. 48 isolated from the vagina, and strain no. 21 which had come from the throat and gave larger colonies. Strains nos. 55 and 51 were chosen as typical $\beta$-strains for the production of antisera. The former had been isolated from the throat and gave smooth growth in fluid and semi-solid media; the latter, isolated from the vagina, gave a more granular growth, although good suspensions were obtained for agglutination. Antisera were also made against the $\gamma$-strains, nos. 27 and 35.

In cross-agglutination tests the antisera to the $\beta$-strains, nos. 51 and 55, agglutinated both homologous and heterologous organisms to a dilution of at least 1/2000. One or other, or both, these antisera agglutinated to a significant titre twenty-one other strains, most of which had previously been identified as $\beta$-strains, the identity of the rest being uncertain. Although with two strains, nos. 33 and 52, the titres were low, the others were agglutinated to at least onequarter of the full titre.

The antiserum to strain no. 48 agglutinated the homologous strain, and strain no. 28 to a dilution of $1 / 1024$ and strains nos. 34 and 46 to $1 / 256$. These four strains with $\alpha$-type colonies had been isolated from the genital tract. They were not agglutinated by the antiserum prepared against strain no. 21 which had been isolated from the throat and had given larger colonies of the $\alpha$-type. This antiserum did not agglutinate any of the other strains. Antisera to the $\gamma$-strains, nos. 27 and 35, agglutinated each other and also two other strains, provisionally identified as $\gamma$, to at least one-quarter of the full titre. In these tests agglutination by at least one antiserum was obtained with all except four strains, suspensions of three of them being unsatisfactory because of granularity. Little cross-agglutination occurred between the $\alpha-\beta$ - and $\gamma$-strains; it did not exceed a dilution of $1 / 64$ and was most often noted with the two $\gamma$-antisera. Sometimes in low dilutions of serum there was a coarse flocculation; this was disregarded as being non-specific. Thus in some tests readings were not obtained in the low dilutions; dilutions of serum less than 1/16 were not tested.

The antisera were tested for their ability to fix complement in the presence of the homologous organism. Strain no. 27 antiserum fixed complement to the same titre as the agglutinating titre; the titres for the other antisera were $1 / 4$ or $1 / 8$ of the agglutinating titres. Complement-fixation tests carried out with a number of the strains confirmed the findings with the agglutination reaction. The genital $\alpha$-strains, the $\beta$ - and $\gamma$-strains and strain no. 21 appeared to be distinct antigenically. Strain no. 27 antiserum fixed complement to 


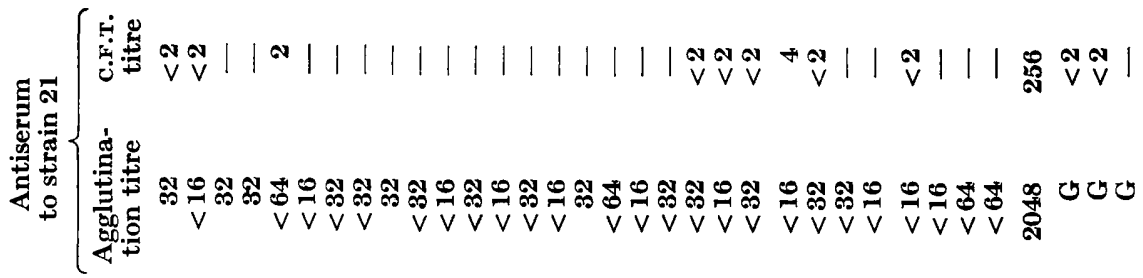

\section{દํำ}

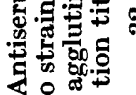

్ㅏㅇㅠ.

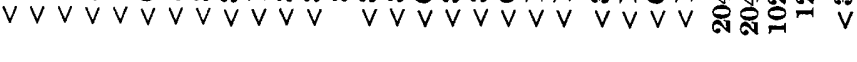
总高 $\checkmark \vee \vee \vee \vee \vee$ $\vec{v} \mathrm{~V}$ ถ ชิ

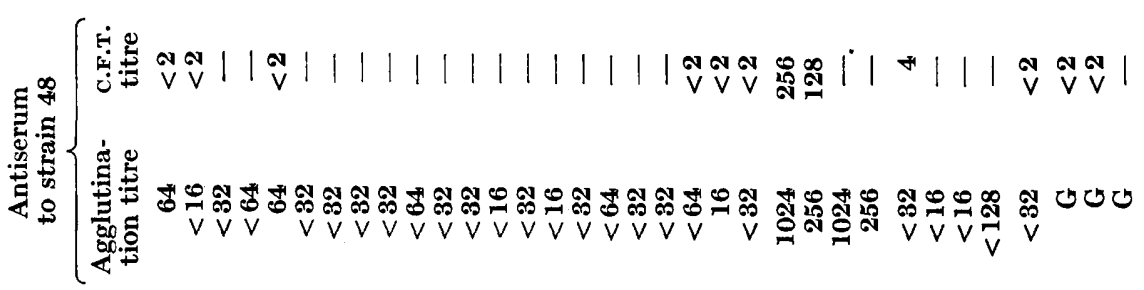

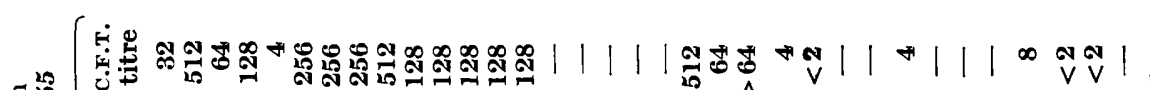
르

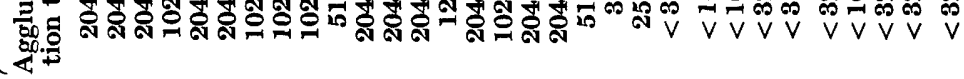

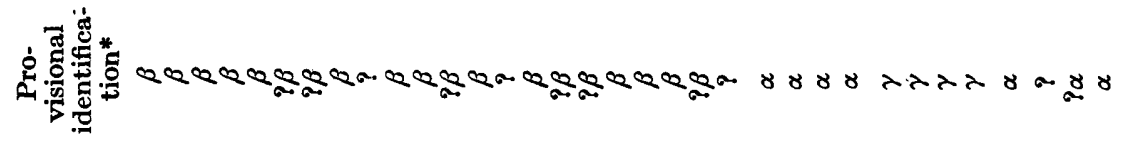

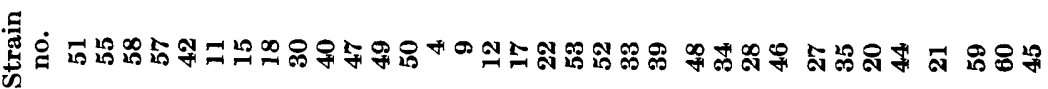


a dilution of $1 / 32$ with strains nos. 55 and 48 , but this was only $\frac{1}{64}$ th of the full titre; otherwise cross-reactions were insignificant. The inagglutinable strain no. 39 was identified as a $\beta$-strain, as it fixed complement to a dilution exceeding 1/64, with strain no. 55 antiserum. Two of the three strains which had been too granular to provide agglutinable suspensions were tested and did not fix complement with any of the antisera.

It has already been noted that not every strain was agglutinated to a full titre by the corresponding antisera. This might be partly explained by poor agglutinability of certain suspensions. However, some $\beta$-strains were agglutinated to full titre by one $\beta$-antiserum and to a low titre only by the other $\beta$-antiserum. As this suggested that antigen differences might occur among the $\beta$-strains, agglutinin-adsorption tests were carried out.

Table 2. Results of agglutinin-adsorption experiments

\begin{tabular}{|c|c|c|c|c|c|c|}
\hline \multirow[b]{2}{*}{ Antigen } & \multicolumn{3}{|c|}{51 antiserum } & \multicolumn{3}{|c|}{55 antiserum } \\
\hline & Unadsorbed & $\begin{array}{c}\text { Adsorbed } \\
\text { with } 55\end{array}$ & $\begin{array}{c}\text { Adsorbed } \\
\text { with } 12\end{array}$ & Unadsorbed & $\begin{array}{l}\text { Adsorbed } \\
\text { with } 51\end{array}$ & $\begin{array}{c}\text { Adsorbed } \\
\text { with } 12\end{array}$ \\
\hline 51 & 2048 & 2048 & 2048 & 2048 & $<64$ & 256 \\
\hline 55 & 2048 & $<32$ & 2048 & 4096 & 4096 & 4096 \\
\hline 12 & 1024 & 256 & $<64$ & 512 & 256 & $<64$ \\
\hline 4 & 128 & 32 & 128 & 512 & 512 & 256 \\
\hline 42 & 2048 & 2048 & 512 & 256 & $<128$ & 128 \\
\hline 17 & 2048 & 1024 & 一 & 1024 & 128 & 128 \\
\hline 15 & 1024 & $<32$ & - & 512 & 256 & 512 \\
\hline
\end{tabular}

Strain no. 51 antiserum after adsorption with strain no. 55, agglutinated the homologous organism to full titre, although no longer capable of agglutinating strain no. 55. Similarly, agglutinins for the homologous organism were not affected by adsorbing strain no. 55 antiserum with strain no. 51. The adsorbed sera were shown to agglutinate a number of other $\beta$-strains, usually to the original titre, but sometimes to a lower titre. Agglutinins for strain no. 15 were removed completely by adsorbing strain 51 antiserum with strain no. 55 . Similar results were obtained by adsorbing the antisera with strain no 12; the adsorbed sera agglutinated the suspensions tested to the original or slightly lower titres. The $\beta$-strains thus appear to have a complex antigenic structure which could not be analysed further.

Although cross-agglutination to approximately full titre occurred between strains nos. 51 and 55, the complement-fixing titre for the heterologous strain was only $\frac{1}{18}$ th that for the homologous. Several other $\beta$-strains in complementfixation tests gave a high titre with one antiserum and a low titre with the other. The complement-fixation test thus appeared to be more specific than the agglutination reaction.

The serological results closely confirmed the original classification of strains based on colonial appearances. All those strains with relatively small colonies, classified as $\alpha$ and isolated from the genital tract, resembled each other antigenically and differed from other strains. Strains with a $\beta$-type of colony, together with a number of strains which had not been definitely identified, 
formed a second antigenic group. Similarly strains classified as $\gamma$ because they produced a characteristic film and precipitate in horse serum media were also alike serologically. Only four strains, isolated from the throat, appeared not to belong to any of these three groups. Strain no. 21 was shown to be antigenically different from the $\alpha$-, $\beta$ - or $\gamma$-strains; the other three strains resembled each other in yielding granular growths in broth cultures and appeared to be unrelated serologically to the other strains. Colonies of strain no. 21 and of one, at least, of the granular strains were of the $\alpha$-type, although larger than those of the genital strains. Thus in future the term ' $\alpha$ ' will be restricted to the group of genital strains.

\section{Cultural characters}

It appeared from previous observations that differences in biological properties could be used for identifying members of the pleuropneumonia group (Edward, 1950 b). These observations were made on laboratory strains which had been maintained by subculture for years. It has now been possible to determine whether an examination of the same properties might assist in the identification of freshly isolated canine strains.

None of the canine strains produced visible growth at room temperature or on media devoid of serum. Representative $\alpha$-, $\beta$ - and $\gamma$-strains grew as well anaerobically, as aerobically. In the earlier examination of members of the pleuropneumonia group the appearance of growth in semi-solid and fluid media had proved most helpful in identification. There was a sharp distinction between those strains which grew in semi-solid media as well-circumscribed irregular masses and in broth as a deposit leaving the supernatant clear, and those which gave a uniform smooth growth. The recently isolated canine strains were more difficult to classify as granular or smooth. Only a few $\beta$-strains, such as strain no. 55, gave a typically smooth growth. The other strains appeared granular, but it was noted that with repeated subculture the tendency to granularity diminished. Many strains were neither definitely granular nor completely smooth. As already noted, the growth of only three strains was so granular that satisfactory agglutinating suspensions could not be obtained.

Fermentation of glucose was not detected with any strain. Haemolysis was noted around colonies on agar plates containing horse serum and horse erythrocytes. There were wide zones of clearing around colonies of $\beta$-strains. Less haemolysis was produced by $\gamma$-strains; only very slight clearing was produced by $\alpha$-strains. The unidentified strains, nos. $21,45,59$ and 60 , were also haemolytic. Reduction of methylene blue was obtained with some, but not all, young cultures of $\beta$ - and $\gamma$-strains. In a few tests reduction by $\alpha$-strains was not noted. Strain no. 21 and the two $\beta$-strains, nos. 51 and 55, grew well on media enriched with rabbit serum instead of horse serum. The $\alpha$-strain, no. 48, and all four $\gamma$-strains tested did not at first grow at all, or growth was very poor, on rabbit serum plates, and a long period of adaptation was necessary before they grew satisfactorily with rabbit serum instead of horse serum.

In these tests no clear-cut differences were obtained between the various strains. Thus tests which had been useful in the identification of other members of the pleuropneumonia group were of no help in differentiating the canine 
strains. Identification of the latter, except by serological means, depended on the colonial appearances already described. The peculiar film and precipitate formed in media containing horse serum distinguished $\gamma$-strains; they were not noted with other strains and were still formed by $\gamma$-strains after maintenance in subculture for months. The characteristic colonial appearances of $\beta$-strains were quickly lost on subculture; with some strains they were less evident even in the first subculture. It was for this reason that a number of $\beta$-strains were not identified with certainty, except by their serological reactions. After subculture the colonies of some $\beta$-strains showed a tendency to vacuolation, so that they resembled $\alpha$-colonies. The $\alpha$-strains maintained their typical colonial appearances for a few subcultures, but strains maintained in this way for months were no longer characteristic.

\section{Distribution of strains among the bitches}

Each animal in the kennels was swabbed once. Pleuropneumonia-like organisms were isolated from approximately half the vaginal swabs and from three-quarters of the throat swabs (Table 3). From three vaginal swabs and from eight throat swabs two different types of organism were isolated; from one vaginal swab three types, $\alpha, \beta$ and $\gamma$, were grown. The number of isolations of each type of organism is shown in Table 3 .

\section{Table 3. Distribution of strains}

\begin{tabular}{|c|c|c|c|c|c|c|c|}
\hline \multirow[b]{3}{*}{ Material } & \multirow{3}{*}{$\begin{array}{c}\text { No. of } \\
\text { samples } \\
\text { examined }\end{array}$} & \multirow{2}{*}{\multicolumn{2}{|c|}{ Samples positive }} & \multicolumn{4}{|c|}{ No. of strains isolated } \\
\hline & & & & & & & \\
\hline & & No. & $\%$ & $\alpha$ & $\beta$ & $\gamma$ & strains \\
\hline Vaginal swabs & 44 & 24 & $\mathbf{5 4 \cdot 5}$ & 10 & 10 & 6 & 2 \\
\hline Throat swabs & 39 & 29 & $74 \cdot 4$ & $\mathbf{0}$ & 23 & 9 & 5 \\
\hline
\end{tabular}

\section{DISCUSSION}

Sabin (1939) and Sabin \& Johnson (1940) showed that several members of the pleuropneumonia group, each with different pathogenic properties, inhabited the upper respiratory tract of mice. Edward $(1950 a)$ found that strains isolated from the genital tract of cattle belonged to one or other of two species, recognized by their cultural properties. The findings in dogs therefore provided another illustration that more than one member of the pleuropneumonia group may reside in the same situation. The different types of canine strain were distinguished by their colonial appearances and, as the differences were well-marked in the primary cultures, it was possible by inspection to establish whether more than one type of organism was present in a culture. Serological investigation largely confirmed the classification of strains based on colonial appearances, and by demonstrating that each type of strain was distinct antigenically suggested that they were separate members of the pleuropneumonia group.

Pleuropneumonia-like organisms were first isolated from dogs by Shoetensack $(1934,1936 a, b)$; they were found in secretion from the upper respiratory tract and in the tissues of animals suffering from canine distemper. Shoetensack 
regarded them as the cause of this disease, and described experiments in which the inoculation of healthy dogs with cultures apparently produced distemper. His strains differed in their properties and appeared to belong to two groups, or species, named by him Asterococcus canis I and A. canis II. It has not been possible to obtain cultures of Shoetensack's strains for comparison with those isolated by us. It is also impossible to determine from his original descriptions whether any of our strains are identical with either $A$. canis I or II. He described an opaque halo surrounding colonies of $A$. canis II on horse serum agar, and it is possible that this was the same as the 'pearly' film and precipitate associated with $\gamma$-strains. Shoetensack, however, stated that an even more marked opacity developed around cultures of $A$. bovis; none of our strains of the latter organism have produced a film or precipitate.

Klieneberger (1938) showed that $A$. canis I and II differed antigenically. She described a dissociation of $A$. canis I into two types of colony, one, the 'coarse', having the characters of the $\alpha$-type. The $\alpha$-strains from the genital tract cannot be identified as $A$. canis I on this evidence alone, because certain antigenically different strains from the throat also had $\alpha$-like colonies. Moreover, after prolonged subculture several other strains showed a tendency to form this coarse type of colony.

The pleuropneumonia group has been classified into a number of species, based on cultural properties, habitat, pathogenicity and antigenic constitution (Sabin, 1941). The differences between the strains isolated by Shoetensack led him to regard them as two different species. Differences between our strains are equally marked and would appear to warrant the assumption that they represent distinct species. Most of these strains belonged to one of three species, provisionally designated by Greek letters to avoid confusion with $A$. canis I and II. An agreed systematic classification and nomenclature for the pleuropneumonia group is very necessary, but should be postponed until more is known about these organisms. We think it is desirable meanwhile to apply provisional labels only to species in order not to prejudice a more systematic nomenclature later.

\section{REFERENCES}

DIENES, L. (1947a). Isolation of pleuropneumonia-like organisms from pathological specimens with the aid of penicillin. Proc. Soc. exp. Biol., N.Y., 64, 165.

Dienes, L. (1947b). Isolation of pleuropneumonia-like organisms from $H$. influenzae with the aid of penicillin. Proc. Soc. exp. Biol., N.Y., 64, 166.

EDWARD, D. G. FF. (1947). A selective medium for pleuropneumonia-like organisms. J. gen. Microbiol. 1, 238.

EDWARD, D. G. FF. (1950a). An investigation of pleuropneumonia-like organisms isolated from the bovine genital tract. J. gen. Microbiol. 4, 4.

EDWARD, D. G. FF. (1950b). An investigation of the biological properties of organisms of the pleuropneumonia group, with suggestions regarding the identification of strains. J. gen. Microbiol. 4, 311.

Edward, D. G. FF. \& Fitzgerald, W. A. (1951). Cholesterol in the growth of organisms of the pleuropneumonia group. J. gen. Microbiol. 5, 576.

Kuieneberger, E. (1938). Pleuropneumonia-like organisms of diverse provenance; some results of an enquiry into methods of differentiation. J.Hyg., Camb., 38, 458. 

Journal of General Microbiology, Vol. 5, No. 3

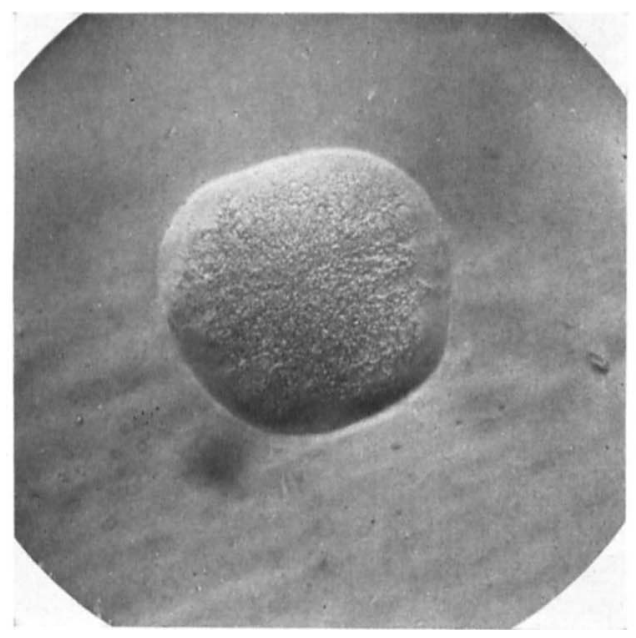

Fig. 1

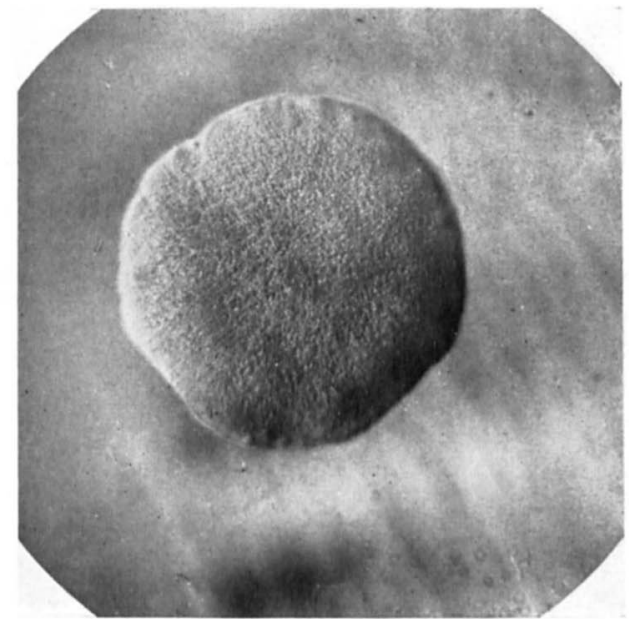

Fig. 2

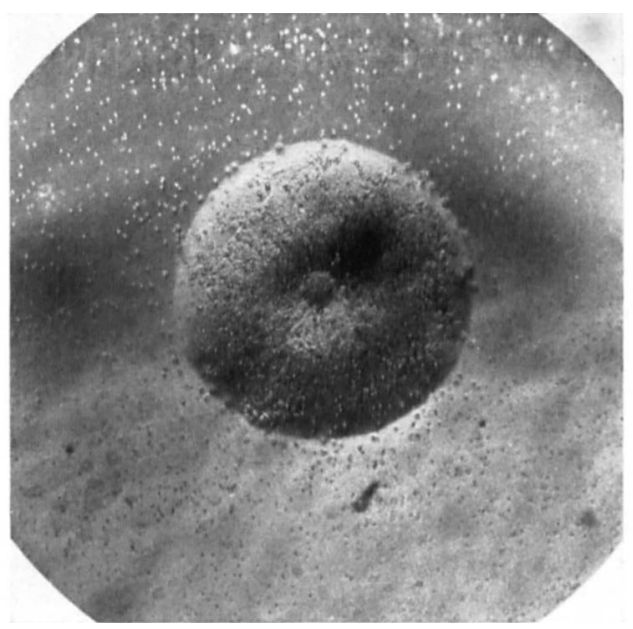

Fig. 3

D. G. Ff. Edward \& W. A. Fitzgerald-Pleuropneumonia-like organisms from dogs. Plate 1 
SABIN, A. B. (1939). Mice as carriers of pathogenic pleuropneumonia-like microorganisms. Science, $90,18$.

SABIN, A. B. (1941). The filtrable micro-organisms of the pleuropneumonia group. Bact. Rev. 5, 1.

SAbin, A. B. \& Johnson, B. (1940). Pathogenic pleuropneumonia-like microorganisms in tissues of normal mice and isolation of new immunological types. Proc. Soc. exp. Biol., N.Y., 44, 569.

Shoetensack, H. M. (1934). Pure cultivation of the filtrable virus isolated from canine distemper. Kitasato Arch. 11, 277.

Shoetensack, H. M. $(1936 a)$. Pure cultivation of filtrable virus isolated from canine distemper (Part II). Morphological and cultural features of Asterococcus canis, Type I, n.sp. and Asterococcus canis, Type II, n.sp. Kitasato Arch. 13, 175.

Shoetensack, H. M. (1936b). Studies concerning the relation between canine distemper and Asterococcus canis, Types I and II. Kitasato Arch. 13, 269.

\section{EXPLANATION OF PLATE}

(Colonies viewed by slightly oblique transmitted light. $\times 100$ approx.)

Fig. 1. Strain 45. Typical $\alpha$-type colony; note coarse marking with vacuolation, the periphery remaining clear.

Fig. 2. Strain 51, a typical $\beta$-strain. Note relatively flattened colony, without an obvious central spot.

Fig. 3. Strain 35, a typical $\gamma$-strain. Dome-shaped colony with well-marked central spot; note the numerous spots in the medium and the beginning of a crinkled film on the surface of the colony. 